\title{
Bone Printing Methods
}

\author{
Filipa Pinto de Oliveira \\ PhD Student Biomedical Engineering, Faculty of Engineering University of Porto, Porto, \\ Portugal; IPO-Porto, Head and Neck Cancer Unit, Porto, Portugal \\ (filipa.pinto.oliveira@gmail.com)
}

\begin{abstract}
In the last few years, three-dimensional printing, that in bone tissue engineering is consider to be a synonymous of additive manufacturing has made its way into the medical field, not only manufacturing medical appliances, study models or building prosthetics. The demand for bone substitution surgeries is growing every year, due to the increase in pathologies affecting bone structure (both traumatic and not traumatic). Nowadays with the possibility of three-dimensional printers becoming bioprinters, engineered bone tissue is starting to become a reality. The aim of this paper is to give the reader an overview of the work done in the last few years towards the advance of three-dimensional printing methods for engineered bone tissue. This paper is divided into six parts, an introduction, then presentation and discussion of the various printing methods with special focus on additive manufacturing (AM), then of bioprinting technologies, further directions of these technologies are considered and a conclusion is done.
\end{abstract}

Author Keywords: Three Dimensional Printing, Additive Manufacturing, Bioprinting, Engineered Bone Tissue, Bone Replacement

Type: Research Article

Open Access Peer Reviewed CC) CC BY

\section{Introduction}

Three dimensional printing (3DP) in the last few years started an ongoing revolution in the medical field in many different aspects that can go from robot helping surgeries to drug delivery systems, customized prosthetics or even tissue and organ fabrication (Ventola 2014). Tissue engineering (TE) according to The Williams Dictionary of Biomaterials is "the persuasion of the body to heal itself through the delivery to the appropriate sites of molecular signals, cells and supporting structures" (Williams 1999). The 3DP of bone is a developing TE that aims to enable regeneration of damaged bone by implantation of biocompatible materials seeded with different types of cell, growth and differentiation factors (Bose, Vahabzadeh, and Bandyopadhyay 2013; Yao et al. 2016; Mandrycky et al. 2016).

Globally 2.2 million of bone grafting surgeries are performed every year due to trauma, osteoporosis, developmental deformities, bone tumours and metastatic disease (Arealis and Nikolaou 2015). Some of these surgeries imply the application of a prosthetic part that is not custom made and needs to be adjusted during the surgical time. The other option is to use grafts and this method involves a second surgery to remove the desired bone from the donor site (autograft) or in the case of allografts it is necessary to make sure that there is immunehistocompatibility between the donor and the recipient (Suárez-Mejías et al. 2015).

The 3DP of bone will help fill the gap in terms of needs of bone grafts with the additional benefit of custom made grafts and only one surgical procedure done to the patient. 
Nowadays the aim of 3DP bone is to mimic as much as possible the bone structure and to make sure that when placed in the defect area the graft will behave as natural bone, creating vascularization, producing bone cells that can normally differentiate into mature bone cells and at the same time have the same resistance to mechanical forces (Luo et al. 2015). In this new field of biofabrication, 3D bioprinting, is defined as the production of complex living and non-living biological products from living cells, biomolecules and biomaterials (Mironov et al. 2009). It is an interdisciplinary technological field that incorporates a variety of fabrication approaches together with cells and developmental biology, mechanical engineering and material sciences.

\section{Printing methods}

By definition 3D printing consists in the fabrication of objects through deposition of material using a printer head, nozzle or another printer technology (often used synonymously with additive manufacturing, especially when associated with low-end machines) (ASTM international committee F42 on Additive manufacturing) (Gu et al. 2016).

The additive manufacturing (AM) is the one most commonly used in printing scaffolds used for bone printing. The reason for this is the technique it possible to print a tailored made piece for a specific patient defect (Kumar et al. 2016) because this procedure permits microengineered scaffolds (Tang et al. 2016). AM technology allows the fabrication of complex shapes needed for this field (Bose, Vahabzadeh, and Bandyopadhyay 2013). The other advantage of this technique is the easy control during scaffold/implant generation, morphology of the exterior in terms of size and shape as well as the control over the internal porous size, although the internal part is not totally controllable yet (Kumar et al. 2016).

\subsection{Additive manufacturing and powder based printing}

Additive manufacturing can be subdivided into three categories according to the type of base used: liquid base (i.e. stereolitography SLA), solid base (i.e. fused deposition modelling FDM) and powder base (i.e. 3D printing) (Munaz et al. 2016).

\subsubsection{Pre printing}

Bone printing needs a specific internal structure in terms of porosity and powder base, 3D printing (powder base) allows this directly from a CAD file (Bose, Vahabzadeh, and Bandyopadhyay 2013). Pre-processing steps, are the group of proceedings that are done before the printing itself. First of all, an image require from the bone defect (X ray, CT scan or $\mathrm{MRI}$ ), then this image needs to be converted into a direct instruction software of the standard template library (STL) for the printing hardware, which includes a series of integrated tools such as automated robotic tools, 3D positioning systems for the printing head, ink reservoir, nozzle systems, video cameras, fibrotic light sources, temperature controllers, piezo electric humidifiers and integrated controlling software (Munaz et al. 2016; Gu et al. 2016). There are a number of steps that are necessary to be checked in the printer before the printing process starts, such as powder packing density, followability and wettability, layer thickness, binder drop volume and saturation (Munaz et al. 2016).

\subsubsection{Printing}

The printing process begins with a roller distributing a predetermined thickness layer of powder from the powder feed supply on to the building chamber. The binder is sprayed from the printer head based on the instructions in the tool path file created according to the CAD file. The viscosity and density of the binder are crucial for the saturation and volume of the drop released from each nozzle. The coordination of powder packing density and drop of binder is crucial for the final printing quality. Each layer that is printed is subjected to a strip 
heater that will make the binder set. This is done repeatedly until all the printing process be finished (Butscher et al. 2011; Munaz et al. 2016; Gu et al. 2016).

\subsubsection{Post printing}

Post processing is the group of steps required to convert the printed structure to an efficient engineered tissue or organ that can be used in the human body (Munaz et al. 2016). This is a crucial process after 3D printing. De-powdering, is the first phase and consists in removing the powder from inside of the structure (through blowing and vibration), this step still needs improvement, because it is very difficult to clean inside microporous structures. Drying is done using air blowing or vibration. It is necessary before curing because it improves the binding strength and facilitates polymerization. The second stage of post processing consists in sintering where the 3D structure is heated, which is crucial to give resistance for the final piece, making the consolidation and transformation into a non-porous solid (i.e. ceramics). Some shrinkage can occur in this phase. Infiltration is another option, a method that also transforms into high-density parts without shrinkage. The only restraint is that the infiltrate material melts at a lower temperature than de 3D printing scaffold, but shrinkage can occur too. Chemical conversion/post hardening (used only for cements/ceramics: calcium phosphate, mg phosphate...) can also be combined with other post printing stages (i.e. sintering), improving the mechanical proprieties of the scaffold (tensile, compressive and flexural strength) and with the advantage of being done at room temperature (Kumar et al. 2016).

\section{Bioprinting considerations}

In the last few years the demand for biomedical scaffolds, has made researches look for biofabrication techniques, that can aid and enhance cell attachment, growth and migration (Tang et al. 2016). So ways to transform the conventional 3D printers into 3D bioprinters have been looked at (Tang et al. 2016; Bose, Vahabzadeh, and Bandyopadhyay 2013; Kumar et al. 2016; Munaz et al. 2016; Gu et al. 2016). Additive manufacturing methodologies can also be used in the construction of scaffolds with living cells (Bose, Vahabzadeh, and Bandyopadhyay 2013). Cell viability and longevity during and after the printing process need to be taken into account (Kumar et al. 2016) but for this to be a reality, conventional 3DP must make adjustments (Gu et al. 2016) so it will be possible to achieve a layer by layer positioning of biomaterials as well as living cells (Munaz et al. 2016) and at the same time to be able to control uniformity of cell distribution or localization in each layer (Tang et al. 2016).

When comparing conventional 3DP and 3D bioprinters it is fair to state that the 3D bioprinters are more complex on account of the materials used (cell/tissue types), growth/differentiation factors needed and sensitivity of construction with living cells (Mandrycky et al. 2016).

There are changes to be done in all of the three stages, pre-printing, printing and post-printing to transform a 3DP into a Bio 3DP (Figure 1). 


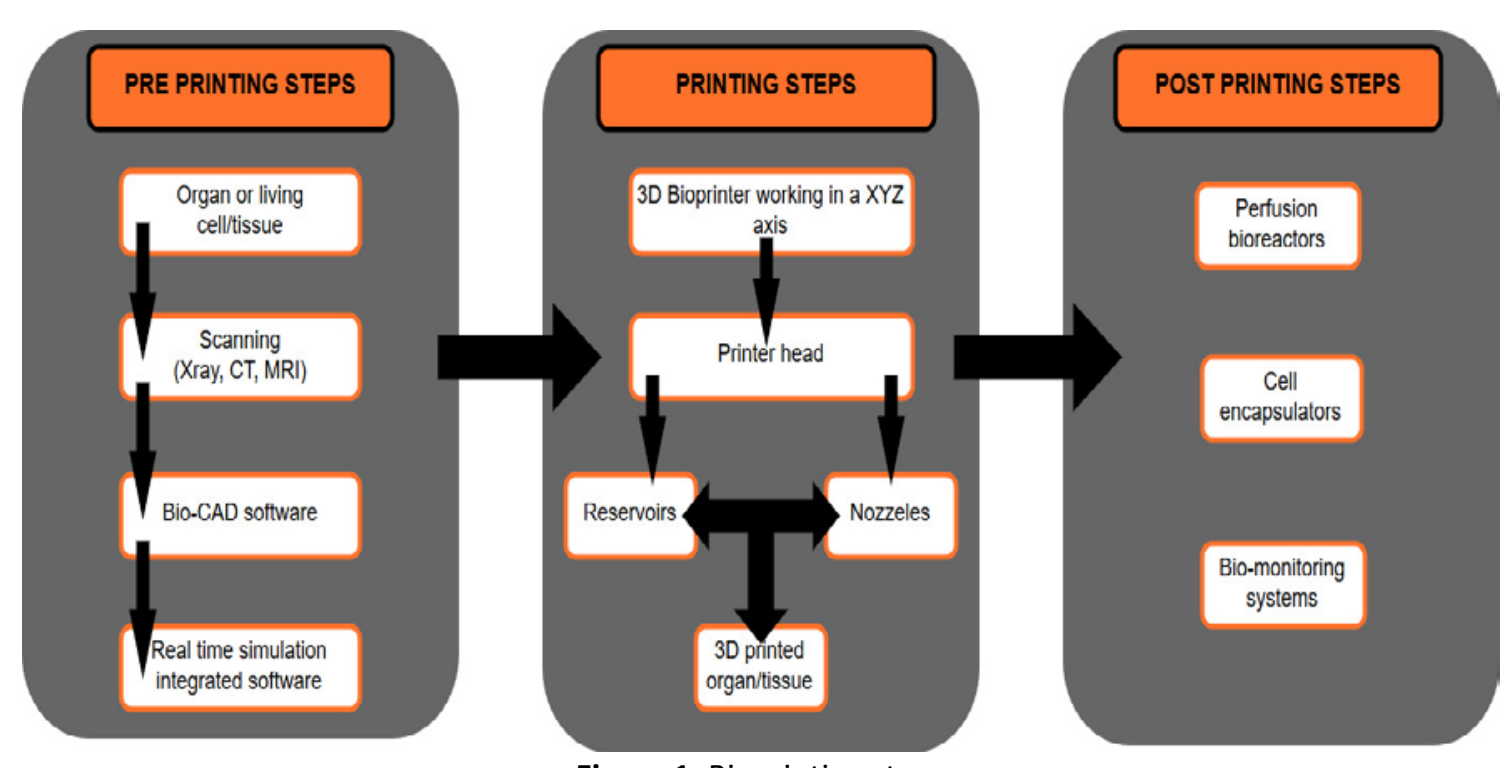

Figure 1: Bioprinting steps

All the figures were made by me making a summary of the content

\subsection{Pre printing}

First of all, medical diagnostic exams, such as X-Ray, magnetic resonance imaging (MRI) or computed tomography (CT) are converted into a bio-computer aid design (Bio-CAD), like MEDCAD or Stryker (Kalamazoo). Bio-CAD software is crucial because it allows visualization of $3 \mathrm{D}$ anatomical structures, differentiates vascular and nerve tissue and generates the desired computational tissue model. Now it is necessary that all the gathered information can be coordinated with the 3D positioning system. This is possible using an integrated and specialized real-time simulation software (i.e. Rhinoceros 4.0) with MATLAB that can turn the bio-CAD design into an exhaustive number of slices with contour boundary paths (Mandrycky et al. 2016). Surface mapping feedback (SMF) is an algorithm-based geometric feedback software that was developed to minimize the accumulation of errors. It compares the bio-CAD model and what is being constructed, and if any errors are found it is possible to adjust them for the subsequent layers. The control of the positioning of the printers head is fundamental to minimize errors in AM, so a positioning system called Bio-assembly tool (BAT) is fundamental for the correct 3D deposit of the different types of cells in each layer (Murphy and Atala 2014). BAT is formed of multiple printers heads that move in XYZ axis, to follow the printing instructions. To avoid inaccuracies in cell placement and in height now a days it is possible to assemble cameras in each printer head to visually control every individual process (Murphy and Atala 2014).

The printer head needs to be modified to be suitable for 3D bioprinting. This has to do with the necessity of multiple materials being used at the same time (i.e. hydrogels, polymers), so a system with multiple nozzles must be used and their operation is adapted according to the material used (i.e. continuous flow, drop on demand or extrusion modes). This all needs to be taken into consideration on account of the use of bio materials because if the cell viability is compromised the final product will not be satisfactory. There are reports that suggest that the cell viability is inversely proportional to the applied force and proportional to the nozzle diameter (Mandrycky et al. 2016; Cui et al. 2012).

The choice of nozzle is vital for 3D bioprinting. The existent nozzles need to be converted into biocompatible ones, this can be achieved by using biocompatible silicones in their interior and exterior. Shape and length are also relevant, conical and short nozzles have shown to have 
better outcomes when cell viability is evaluated, when compared to cylindrical and longer nozzles (Mandrycky et al. 2016).

The ink for a 3D bioprinting process, must be biocompatible while permitting cell adhesion and proliferation, should have a low viscosity that allows a good flow from the printers head and after the 3D printing needs to be stiff and have strength to maintain 3D structure (Mandrycky et al. 2016) (Figure 2). For building scaffolds the bio ink used must have these three proprieties, so the cells seeded after the 3D bioprinting, can grow and behave as in their natural environment.

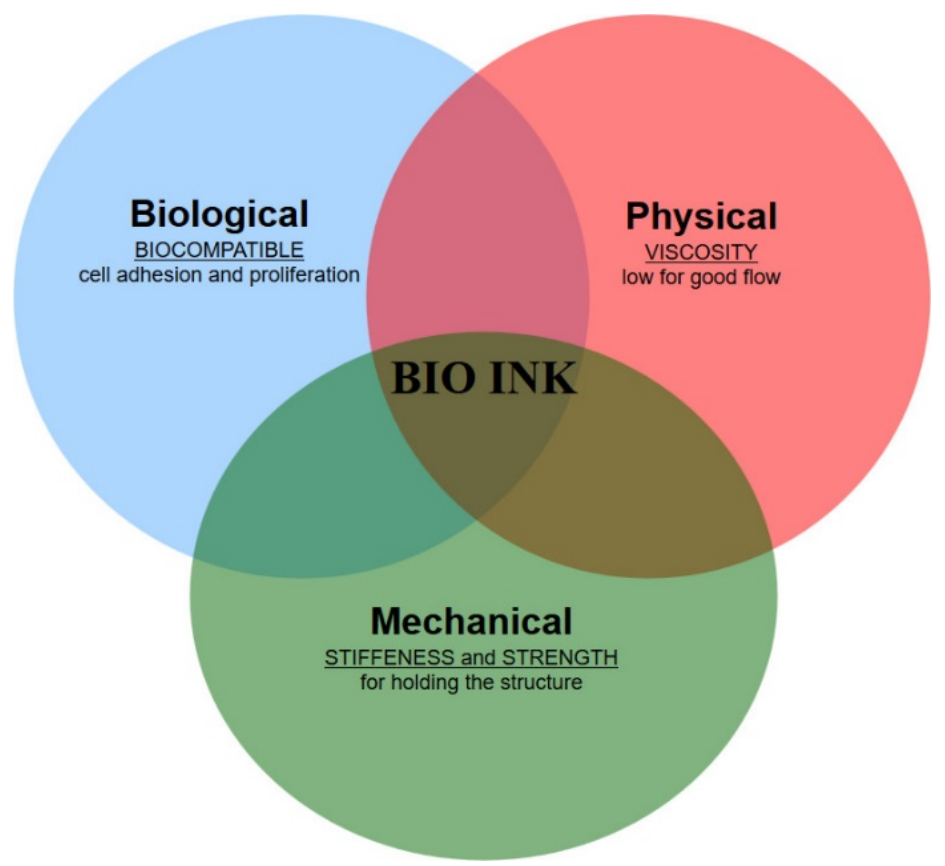

Figure 2: Target proprieties of bio ink materials

All the figures were made by me making a summary of the content

\subsection{2 Printing}

This step is the actual printing session of the bio ink using the bioprinters. Clinical cell sorters (e.g. celution, cytori therapeutics) are used along with, cell propagation bioreactors (e.g. Aastrom Bioscience) and cell differentiators to construct the desired biological structures and maintain the cells alive (Mandrycky et al. 2016; Chen et al. 2016).

Another important mechanism in this step is the 3D positioning system and a control software, that can work together or separately but they always have to work synchronized, so that the printer heads with their microcontroller unit and multiple nozzles are controlled both spatial and temporal, so the type of cell is selected correctly and delivered in the exact time to the exact place (Singh, Singh, and Han 2016). If this system is not working correctly the end result will not be a satisfactory tissue engineered bone because it may have problems in the structure that will lead to poor support or even in the unviability of cell seeding (Jakob et al. 2013).

\subsection{Post printing}

This is the group of necessary steps to convert the printed structure to an efficient engineered tissue or organ that can be used in the human body. When bone is damaged, the body will recognize the damaged area and start the healing process. This means that a number of mechanisms are activated. Cells for tissue repair and development are stimulated and recruited to the injured site, this will include signalling molecules, matrix chemistry and mechanical forces (Dawson et al. 2014; Valenti, Carbonare, and Mottes 2017). 
This stage aims to make sure that all the cells and signalling molecules that were printed previously continue to stay in the conditions that will mimic natural bone tissue. For this to happen it is necessary to include perfusion bioreactors, cell encapsulators and bio-monitoring systems. All of these auxiliary machines have their own important roles in bioprinting (Munaz et al. 2016; Mandrycky et al. 2016).

\section{Bioprinting technologies}

There are three techniques used in for depositing and patterning biomaterials, that are also used in bone engineering using additive manufacturing (Luo et al. 2015; Butscher et al. 2011; Munaz et al. 2016). Microextrusion deposition method (Munaz et al. 2016; Butscher et al. 2011; Tang et al. 2016) is known as pressure-assisted bioprinting (PAB) too (Li et al. 2016). Pastes, solutions or dispersions are the biomaterials used for this printing method (Chia and Wu 2015). A thermal source heats a thermal filament that makes a motion of pneumatic pressure into the bio ink to come out of the nozzles (Li et al. 2016) that will form the 3D structure layer-by-layer. The previous layer acts as subtract for the next one (Luo et al. 2015; Scawn et al. 2015; Bryers, Giachelli, and Ratner 2012). The great advantage of this technique is that it allows to be done at room temperature and permits that the cells are printed directly achieving an identical distribution (Li et al. 2016).

The Laser-assisted cell printing_(LAB), uses a pulsatile laser source, a receiving substrate for patterning and assembling cells. A ribbon coated with biomaterials uses a laser absorvant interlayer, and this heat is transferred to the bio ink. During this process, the laser irradiates the ribbon and makes the bio ink to be transformed into drops (Li et al. 2016). The cells individually are placed by the laser in the solid surface (substrate). This meticulous process lets, in theory, the construction of cellular micropatterns (Luo et al. 2015; Scawn et al. 2015; Mandrycky et al. 2016), from -pico to -micro depending on the following thickness of the bio ink, material proprieties, energy of the laser pulse, wettability of the substrate, organization of the structure and printing speed (Li et al. 2016; Guillemot et al. 2010a; Guillemot et al. 2010b).

Inkjet-based cell printing, is a biotechnology that was inspired on the conventional inkjet printing process (Li et al. 2016). They can be drop-on-demand or continuous ejection (Zhou 2016). The advantages of an inkjet printer is to be low-price, high resolution and speed. They are simple to use and widely available, simple components, ready-to-use design and intuitive software when compared to others (Zhou 2016). Multiple printer heads with different cell types can be used at the same time allowing microscale multicellular organizations (Mandrycky et al. 2016; Scawn et al. 2015; Luo et al. 2015). The printing process is computer control without any contact between the bio ink and the hydrogel or the culture dish. There are two main methods for the bio ink to be dropped: using piezoelectric technology or a thermal source, both have some concerns related to cell viability. When using piezoelectric technology, a transient pressure is formed and creates the drops. This method has the advantage of less nozzle blocking ( $L i$ et al. 2016) creating a more even and level pattern (Li et al. 2016; Nakamura et al. 2005; Saunders, Gough, and Derby 2008). But the great advantage of this method is that a cellular viability up to $90 \%$ has been reported (Nakamura et al. 2005).The second process uses thermal source that will make the bio ink come out of the nozzle. However this method has been largely used and it is not expensive, it is difficult to have an even printing process. On one hand, it is fairly normal for the nozzle to block or the droplets can be mixed or placed unevenly, on the other hand the main problem is that the bio ink components (cells and proteins) can easily be damaged by the heat (Boland et al. 2006; Cui et al. 2010; Murphy and Atala 2014; Cui et al. 2012; Jana and Lerman 2015). 


\section{Further directions}

In the future with the availability of these technologies, bone TE could be an alternative to the available grafting methods (autografts and allografts), moreover the level of stress for the operating team can be reduced as can risks during surgery and the quality of life of the patient will be improve.

First, surgical planning can be done more accurately, that will diminish the margin of error, the need for adaptation of the prosthetic bone tend to be less needed, all contributing for the reduction of the operating time. Patient quality of life will improve not only because the bone that needs to be replaced will be custom made but also because this can all be done only in one medical intervention (no need for a second surgery) (Suárez-Mejías et al. 2015). To achieve what was previously a number of limitations must still be overcome and many improvements are still necessary in bone TE. The aim of o bone TE is for it to be osseointegrated into donor site and for this to become a reality, it is essential for the vascularization be high (Shadjou and Hasanzadeh 2015; Fedorovich et al. 2011), the infection rate needs to be low (Tang et al. 2016), the strength needs to be high and the degradation rate needs to be low too (Liu, Lim, and Teoh 2013). When all of these four main factors achieve their best performance engineered bone TE grafts will be a complete success (Figure 3 ).

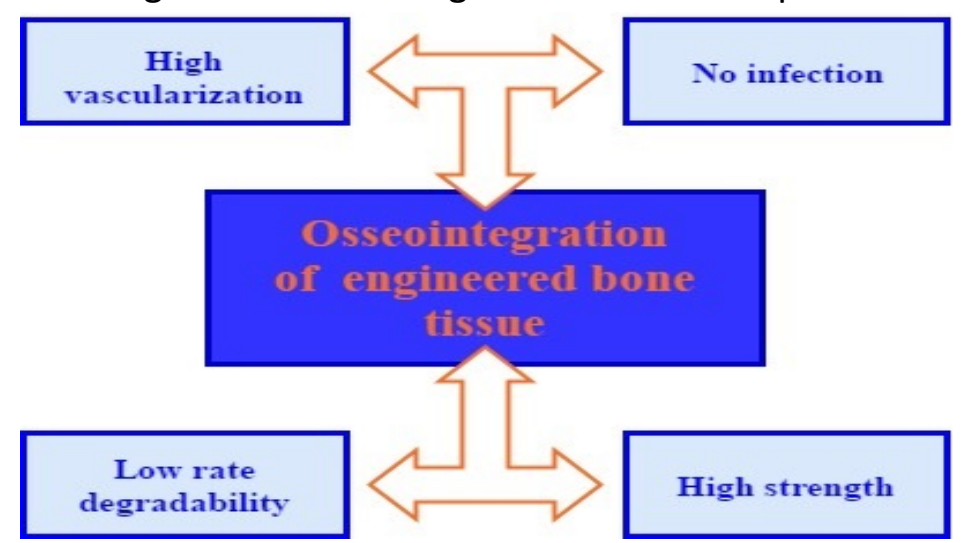

Figure 3: Factors that have a direct influence in the osseointegration of engineered bone tissue in the human body

All the figures were made by me making a summary of the content

\section{Conclusion}

The aim of this paper is to give an overview of 3D bone printing technologies, limitations and future directions. Additive manufacturing is the technology of choice because it allows fine detailed architecture that is mostly necessary when printing bone.

Nowadays there is a demand for 3D bioprinters and it is possible to say that the adjustments in 3D conventional printers can be done for them to work biologically. This modifications are not only done in the printer structure (nozzles, reservoirs and printer head) but also in the process of printing and in the bio ink. After printing is also a crucial phase, because it is mandatory that all the mechanisms for cell seeding, integration and proliferation are working perfectly. New bio inks need to be developed to ensure that they are easily printable, allowing controllable and reproducible printing of cells and have some solidification mechanism to enable the fabrication of 3D structures. The difficulty in this, it is that mechanical and physical proprieties (strength and degradability) are of high importance in 3DP bone printing but it is important to be balanced with that fact that cells are living organisms that cannot be damaged. 
Looking into the future there are new techniques that still require some development but the bioprinting technologies already developed can be used in combination in order to achieve osseointegration of 3D bone printing.

\section{References}

Arealis, G. and V. S. Nikolaou. 2015. "Bone printing: New frontiers in the treatment of bone defects". Injury 46 Suppl 8:S20-22. Accessed March 18, 2017. DOI: 10.1016/S00201383(15)30050-4.

Boland, T., T. Xu, B. Damon and X. Cui. 2006. "Application of inkjet printing to tissue engineering". Biotechnology Journal 1 (9):910-917. Accessed April 1, 2017. DOI: 10.1002/biot.200600081.

Bose, Susmita, Sahar Vahabzadeh and Amit Bandyopadhyay. 2013. "Bone tissue engineering using 3D printing". Materials Today 16 (12):496-504. Accessed March 18, 2017. DOI: 10.1016/j.mattod.2013.11.017.

Bryers, J. D., C. M. Giachelli and B. D. Ratner. 2012. "Engineering biomaterials to integrate and heal: The biocompatibility paradigm shifts". Biotechnology and Bioengineering 109 (8):1898-1911. Accessed April 1, 2017. DOI: 10.1002/bit.24559.

Butscher, A., M. Bohner, S. Hofmann, L. Gauckler and R. Müller. 2011. "Structural and material approaches to bone tissue engineering in powder-based three-dimensional printing". Acta Biomaterialia 7 (3):907-920. Accessed March 18, 2017. DOI: 10.1016/j.actbio.2010.09.039.

Chen, Hu, Xu Yang, Litong Chen, Yong Wang and Yuchun Sun. 2016. "Application of FDM threedimensional printing technology in the digital manufacture of custom edentulous mandible trays". Scientific Reports 6:19207. Accessed April 1, 2017. DOI: 10.1038/srep19207.

Chia, H. N. and B. M. Wu. 2015. "Recent advances in 3D printing of biomaterials". Journal of Biological Engineering 9 (1):4. Accessed March 18, 2017. DOI: 10.1186/s13036-015-0001-4.

Cui, X., D. Dean, Z. M. Ruggeri and T. Boland. 2010. "Cell damage evaluation of thermal inkjet printed Chinese hamster ovary cells". Biotechnology and Bioengineering 106 (6):963-969. Accessed March 18, 2017. DOI: 10.1002/bit.22762.

Cui, X., T. Boland, D. D. D'Lima and M. K. Lotz. 2012. "Thermal inkjet printing in tissue engineering and regenerative medicine". Recent Patents on Drug Delivery \& Formulation 6 (2):149-155. Accessed March 18, 2017. DOI: 10.2174/187221112800672949.

Dawson, Jonathan I., Janos Kanczler, Rahul Tare, Moustapha Kassem and Richard O. C. Oreffo. 2014. "Concise review: Bridging the gap: Bone regeneration using skeletal stem cell-based strategies - Where are we now?". STEM CELLS 32 (1):35-44. Accessed March 20, 2017. DOI: 10.1002/stem.1559.

Fedorovich, Natalja E., Jacqueline Alblas, Wim E. Hennink, F. Cumhur Öner and Wouter J. A. Dhert. 2011. "Organ printing: The future of bone regeneration?". Trends in Biotechnology 29 (12):601-606. Accessed March 18, 2017. DOI: 10.1016/j.tibtech.2011.07.001.

Gu, Bon Kang, Dong Jin Choi, Sang Jun Park, Min Sup Kim, Chang Mo Kang and Chun-Ho Kim. 2016. "3-dimensional bioprinting for tissue engineering applications". Biomaterials Research 20 (1):12. Accessed April 1, 2017. DOI: 10.1186/s40824-016-0058-2.

Guillemot, F., A. Souquet, S. Catros and B. Guillotin. 2010a. "Laser-assisted cell printing: Principle, physical parameters versus cell fate and perspectives in tissue engineering". Nanomedicine 5 (3):507-515. Accessed April 1, 2017. DOI: 10.2217/nnm.10.14.

Guillemot, F., A. Souquet, S. Catros, B. Guillotin, J. Lopez and M. Faucon. 2010b. "Highthroughput laser printing of cells and biomaterials for tissue engineering". Acta 
Biomaterialia 6 (7):2494-2500. Accessed March 18, 2017. DOI: 10.1016/j.actbio.2009.09.029.

Jakob, F., R. Ebert, A. Ignatius, T. Matsushita, Y. Watanabe, J. Groll and H. Walles. 2013. "Bone tissue engineering in osteoporosis". Maturitas 75 (2):118-124. Accessed March 18, 2017. DOI: 10.1016/j.maturitas.2013.03.004.

Jana, S. and A. Lerman. 2015. "Bioprinting a cardiac valve". Biotechnology Advances 33 (8):1503-1521. Accessed March 20, 2017. DOI: 10.1016/j.biotechadv.2015.07.006.

Kumar, Alok, Sourav Mandal, Srimanta Barui, Ramakrishna Vasireddi, Uwe Gbureck, Michael Gelinsky and Bikramjit Basu. 2016. "Low temperature additive manufacturing of three dimensional scaffolds for bone-tissue engineering applications: Processing related challenges and property assessment". Materials Science and Engineering: R: Reports 103:139. Accessed March 18, 2017. DOI: 10.1016/j.mser.2016.01.001.

Li, Jipeng, Mingjiao Chen, Xianqun Fan and Huifang Zhou. 2016. "Recent advances in bioprinting techniques: Approaches, applications and future prospects". Journal of Translational Medicine 14 (1):271. Accessed April 1, 2017. DOI: 10.1186/s12967-016-1028-0.

Liu, Yuchun, Jing Lim and Swee-Hin Teoh. 2013. "Review: Development of clinically relevant scaffolds for vascularised bone tissue engineering". Biotechnology Advances 31 (5):688705. Accessed March 20, 2017. DOI: 10.1016/j.biotechadv.2012.10.003.

Luo, Yongxiang, Dong Zhai, Zhiguang Huan, Haibo Zhu, Lunguo Xia, Jiang Chang and Chengtie Wu. 2015. "Three-dimensional printing of hollow-struts-packed bioceramic scaffolds for bone regeneration". ACS Applied Materials \& Interfaces 7 (43):24377-24383. Accessed March 20, 2017. DOI: 10.1021/acsami.5b08911.

Mandrycky, Christian, Zongjie Wang, Keekyoung Kim and Deok-Ho Kim. 2016. "3D bioprinting for engineering complex tissues". Biotechnology Advances 34 (4):422-434. Accessed April 1, 2017. DOI: 10.1016/j.biotechadv.2015.12.011.

Mironov, V., T. Trusk, V. Kasyanov, S. Little, R. Swaja and R. Markwald. 2009. "Biofabrication: A 21st century manufacturing paradigm". Biofabrication 1 (2):022001. Accessed April 1, 2017. DOI: 10.1088/1758-5082/1/2/022001.

Munaz, Ahmed, Raja K. Vadivelu, James St. John, Matthew Barton, Harshad Kamble and NamTrung Nguyen. 2016. "Three-dimensional printing of biological matters". Journal of Science: Advanced Materials and Devices 1 (1):1-17. Accessed March 18, 2017. DOI: 10.1016/j.jsamd.2016.04.001.

Murphy, S. V. and A. Atala. 2014. "3D bioprinting of tissues and organs". Nature Biotechnology 32 (8):773-785. Accessed March 20, 2017. DOI: 10.1038/nbt.2958.

Nakamura, M., A. Kobayashi, F. Takagi, A. Watanabe, Y. Hiruma, K. Ohuchi, Y. Iwasaki, M. Horie, I. Morita and S. Takatani. 2005. "Biocompatible inkjet printing technique for designed seeding of individual living cells". Tissue Engineering 11 (11-12):1658-1666. Accessed March 20, 2017. DOI: 10.1089/ten.2005.11.1658.

Saunders, R. E., J. E. Gough and B. Derby. 2008. "Delivery of human fibroblast cells by piezoelectric drop-on-demand inkjet printing". Biomaterials 29 (2):193-203. Accessed March 18, 2017. DOI: 10.1016/j.biomaterials.2007.09.032.

Scawn, Richard L., Alex Foster, Bradford W. Lee, Don O. Kikkawa and Bobby S. Korn. 2015. "Customised 3D printing: An innovative training tool for the next generation of orbital surgeons". Orbit 34 (4):216-219. Accessed March 22, 2017. DOI: 10.3109/01676830.2015.1049367. 
Shadjou, N. and M. Hasanzadeh. 2015. "Bone tissue engineering using silica-based mesoporous nanobiomaterials: Recent progress". Materials Science and Engineering: $C$ 55:401-409. Accessed April 5, 2017. DOI: 10.1016/j.msec.2015.05.027.

Singh, Deepti, Dolly Singh and Sung Han. 2016. "3D printing of scaffold for cells delivery: Advances in skin tissue engineering". Polymers 8 (1):19. Accessed April 1, 2017. DOI: 10.3390/polym8010019.

Suárez-Mejías, Cristina, Gorka Gomez-Ciriza, Israel Valverde, Carlos Parra Calderón and Tomás Gómez-Cía. 2015. "New technologies applied to surgical processes: Virtual reality and rapid prototyping". Studies in Health Technology and Informatics 210:669-671. Accessed April 2, 2017. http://europepmc.org/abstract/MED/25991234.

Tang, D., R. S. Tare, L. Y. Yang, D. F. Williams, K. L. Ou and R. O. Oreffo. 2016. "Biofabrication of bone tissue: Approaches, challenges and translation for bone regeneration". Biomaterials 83:363-382. Accessed March 22, 2017. DOI: 10.1016/j.biomaterials.2016.01.024.

Valenti, Maria, Luca Dalle Carbonare and Monica Mottes. 2017. "Osteogenic differentiation in healthy and pathological conditions". International Journal of Molecular Sciences 18 (1):41. Accessed March 18, 2017. DOI: 10.3390/ijms18010041.

Ventola, C. L. 2014. "Medical applications for 3D printing: Current and projected uses". P \& T: A Peer-Reviewed Journal for Formulary Management 39 (10):704-711. Accessed March 7, 2017. https://www.ncbi.nlm.nih.gov/pmc/articles/PMC4189697.

Williams, D. F. 1999. The Williams dictionary of biomaterials. Vol. 1. Liverpool: Liverpool University Press.

Yao, R., G. Xu, S. S. Mao, H. Y. Yang, X. T. Sang, W. Sun and Y. L. Mao. 2016. "Three-dimensional printing: Review of application in medicine and hepatic surgery". Cancer Biology \& Medicine 13 (4):443-451. Accessed March 19, 2017. DOI: 10.20892/j.issn.20953941.2016.0075.

Zhou, Y. 2016. "The application of ultrasound in 3D bioprinting". Molecules 21 (5):590. Accessed March 19, 2017. DOI: 10.3390/molecules21050590. 